TRANSACTIONS OF THE

AMERICAN MATHEMATICAL SOCIETY

Volume 353 , Number 12, Pages 4779-4800

S 0002-9947(01)02833-1

Article electronically published on June 21, 2001

\title{
RANDOM VARIABLE DILATION EQUATION AND MULTIDIMENSIONAL PRESCALE FUNCTIONS
}

\author{
JULIE BELOCK AND VLADIMIR DOBRIC
}

\begin{abstract}
A random variable $Z$ satisfying the random variable dilation equation $M Z \stackrel{d}{=} Z+G$, where $G$ is a discrete random variable independent of $Z$ with values in a lattice $\Gamma \subset \mathbf{R}^{d}$ and weights $\left\{c_{k}\right\}_{k \in \Gamma}$ and $M$ is an expanding and $\Gamma$-preserving matrix, if absolutely continuous with respect to Lebesgue measure, will have a density $\varphi$ which will satisfy a dilation equation

$$
\varphi(x)=|\operatorname{det} M| \sum_{k \in \Gamma} c_{k} \varphi(M x-k) .
$$

We have obtained necessary and sufficient conditions for the existence of the density $\varphi$ and a simple sufficient condition for $\varphi$ 's existence in terms of the weights $\left\{c_{k}\right\}_{k \in \Gamma}$. Wavelets in $\mathbf{R}^{d}$ can be generated in several ways. One is through a multiresolution analysis of $L^{2}\left(\mathbf{R}^{d}\right)$ generated by a compactly supported prescale function $\varphi$. The prescale function will satisfy a dilation equation and its lattice translates will form a Riesz basis for the closed linear span of the translates. The sufficient condition for the existence of $\varphi$ allows a tractable method for designing candidates for multidimensional prescale functions, which includes the case of multidimensional splines. We also show that this sufficient condition is necessary in the case when $\varphi$ is a prescale function.
\end{abstract}

\section{INTRODUCTION}

Multiresolution analysis on $\mathbf{R}^{d}$ is one possible framework for construction of wavelet bases. Let $\Gamma$ be a lattice in $\mathbf{R}^{d}$ and let $M: \mathbf{R}^{d} \rightarrow \mathbf{R}^{d}$ be an expansive linear transformation, that is, all eigenvalues of $M$ have modulus greater than 1 , such that $M \Gamma \subseteq \Gamma$. Then $m=|\operatorname{det} M|$ is an integer, greater than one, equal to the order of the group $\Gamma / M \Gamma$. A multiresolution analysis associated to $\Gamma$ and $M$ with prescale function $\varphi$ is an increasing sequence of subspaces of $L^{2}\left(\mathbf{R}^{d}\right)$, $\cdots \subseteq V_{-1} \subseteq V_{0} \subseteq V_{1} \subseteq \cdots$ satisfying the following four conditions:

(i) $\bigcup V_{j}$ is dense in $L^{2}\left(\mathbf{R}^{d}\right)$; $j$

(ii) $\bigcap_{j} V_{j}=\{0\}$;

(iii) $f(\cdot) \in V_{j} \Leftrightarrow f\left(M^{-j}.\right) \in V_{0}$;

(iv) $\{\varphi(\cdot-\gamma)\}_{\gamma \in \Gamma}$ is a Riesz basis for $V_{0}$.

Received by the editors January 10, 2000 and, in revised form, January 8, 2001.

2000 Mathematics Subject Classification. Primary 60A10, 60G50; Secondary 42C40, 42C15. Key words and phrases. Dilation equations, tilings, wavelets.

(C)2001 American Mathematical Society 
A wavelet basis associated to the multiresolution analysis is an orthonormal basis for $L^{2}\left(\mathbf{R}^{d}\right)$ of the form $\left\{m^{j / 2} \psi_{k}\left(M^{j} \cdot-\gamma\right): j \in Z, \gamma \in \Gamma, 1 \leq k \leq m\right\}$ where

$$
\psi_{k}(x)=\sum_{\gamma \in \Gamma} a_{k}(\gamma) \varphi(M x-\gamma)
$$

and $\left\{a_{k}(\gamma)\right\}_{\gamma \in \Gamma}$ is square summable for $1 \leq k \leq m$. The functions $\left\{\psi_{k}\right\}_{k=1}^{m}$ are called the wavelet generators. When the lattice translates of $\varphi$ form an orthonormal basis of $V_{0}$ we take $\psi_{1}:=\varphi$.

Conditions (iii) and (iv) together imply that the set $\{\varphi(M \cdot-\gamma)\}_{\gamma \in \Gamma}$ is a Riesz basis for the subspace $V_{1}$. Since $\varphi \in V_{0} \subseteq V_{1}$, we can write

$$
\varphi(x)=\sum_{\gamma \in \Gamma} a(\gamma) \varphi(M x-\gamma) ;
$$

equation (1.1) is called a dilation equation.

One way to understand (1.1) is through a probabilistic approach. Consider a discrete random variable $G$ with values in a subset $\Gamma_{1}$ of $\Gamma$ and a random variable $Z$, independent of $G$, with values in $\mathbf{R}^{d}$, both defined on a complete probability space $(\Omega, \mathcal{F}, P)$, which satisfy

$$
M Z \stackrel{d}{=} Z+G
$$

Here, $\stackrel{d}{=}$ denotes equality of the corresponding laws. Assume that $Z$ is absolutely continuous with respect to Lebesgue measure and denote its density by $\varphi$. Equation (1.2) implies that $\varphi$ satisfies the dilation equation (1.1) with $a(\gamma)=$ $|\operatorname{det} M| P(G=\gamma)$. Our approach to constructing candidates for prescale functions comes from understanding the structure of the solution of this random variable dilation equation.

In the one-dimensional case with $M=2$, Gundy and Zhang [6] proved that $Z$ is absolutely continuous with respect to Lebesgue measure if and only if the fractional part of $Z$ is uniform. They also gave a sufficient condition for the uniformity of the fractional part. In the higher dimensional case, we show that the statements of Gundy and Zhang hold true when a proper notion of the "fractional" part of a random variable is introduced. We have found the theory of self-affine tilings of $\mathbf{R}^{d}$ and use of the digit representation of the fractional part of $Z$ to be the correct framework for the higher dimensional case. The major difficulty in generalizing the results to higher dimensions comes from the fact that $M$ may not be merely an expansion but may include a rotation. Such an $M$ causes a tile to have, in general, a fractal boundary. The boundary difficulties called for some new techniques of proofs beyond those used in [6].

In Section 2 we introduce notation needed to express an explicit solution $Z$ to (1.2). Definitions of the "fractional" and "integer" parts of an $\mathbf{R}^{d}$-valued random variable $Z$ are given based on concepts of self-affine tilings. We also give some basic results regarding the fractional part of $Z$. In Section 3 we give necessary and sufficient conditions under which the random variable $Z$ will have a density, in terms of the fractional part of $Z$. In Section 4 we give a simple sufficient condition on the weights on the values of $G$ which guarantee absolute continuity of $Z$. In Section 5 we give examples of density functions obtained using these results. In Section 6 we show that the sufficient condition of Section 4 is also necessary when $\varphi$ is a prescale function. 


\section{BASIC PROPERTIES OF A RANDOM VARIABLE DILATION EQUATION SOLUTION}

In order to write an explicit solution of (1.2), some definitions are needed. Let $G_{1}, G_{2}, \ldots$ be an i.i.d. sequence of random variables defined on the space $(\Omega, \mathcal{F}, P)$, with $G_{1} \stackrel{d}{=} G$. Recall that $G$ is discrete with values in the lattice. Assume

$$
\sum_{j=1}^{\infty} M^{-j} G_{j}<\infty \text { a.s. }
$$

Then the sequence $\left\{Z_{k}\right\}$ defined by

$$
Z_{k}=\sum_{j=1}^{\infty} M^{-j} G_{j+k} \text { for } k=1,2, \ldots
$$

is a sequence of random variables. Note that the following two properties hold:

$$
M Z_{k}=M\left(\sum_{j=1}^{\infty} M^{-j} G_{j+k}\right)=G_{k+1}+\sum_{j=2}^{\infty} M^{-j+1} G_{j+k}=G_{k+1}+Z_{k+1}
$$

and

$$
Z_{0} \stackrel{d}{=} Z_{k} \text {, and } G_{k} \text { is independent of } Z_{k} .
$$

Therefore for any $k, Z_{k}$ solves the dilation equation (1.2).

The fractional part of $Z$ will play an essential role in what follows. In order to define the fractional part of $Z$, we first invoke some basic facts about self-affine tilings. Let $\Gamma_{0}$ denote a set of coset representatives of $\Gamma / M \Gamma$, and without loss of generality, we assume $0 \in \Gamma_{0}$. A self-affine tiling of $\mathbf{R}^{d}$ consists of a closed set $T$ with nonempty interior such that

$$
\bigcup_{\gamma \in \Gamma}(T+\gamma)=\mathbf{R}^{d} \text { and } \bigcup_{\gamma \in \Gamma_{0}}(T+\gamma)=M T .
$$

Clearly a tiling depends on the choice of $\Gamma_{0}$. In dimensions $d=2$ and 3 , one can always find a $\Gamma_{0}$ that admits a self-affine tiling, and in higher dimensions it can be done for $m=|\operatorname{det} M|>d[10]$. For the remainder of the paper, we will assume that $\Gamma_{0}$ admits a self-affine tiling.

The lattice translates of the interior of $T$ are disjoint and $\operatorname{int} T \neq \varnothing[1$, so if $x \in \bigcup_{\gamma \in \Gamma}($ int $T+\gamma)$, then $x \in$ int $T+\gamma_{x}$ where $\gamma_{x}$ denotes the unique element of $\Gamma$ giving the location of the point $x$. If $x \notin \bigcup_{\gamma \in \Gamma}(i n t T+\gamma)$, then we say $x$ is a boundary point and note that $x \in \bigcap_{\gamma \in \Gamma_{1}}(T+\gamma)$, for some finite $\Gamma_{1} \subseteq \Gamma$. The fact that $\Gamma_{1}$ is finite follows from the compactness of $T$.

Define [.] : $\mathbf{R}^{d} \rightarrow \Gamma$ by

$$
[x]=\left\{\begin{aligned}
& \gamma_{x} \text { if } x \in \bigcup_{\gamma \in \Gamma}(\text { int } T+\gamma) \\
& \max _{\gamma \in \Gamma_{1}} \gamma \text { if } x \text { is a boundary point }
\end{aligned}\right.
$$

where "max" is meant in the sense of the dictionary ordering of $\mathbf{R}^{d}$.

Proposition 1. [·] is Borel-measurable. 
Proof. We only need to consider $\{x \mid[x]=\gamma\}$ for a fixed $\gamma \in \Gamma$. Since $T$ is compact and $\Gamma$ is countable,

$$
\left[\gamma_{1}\right]^{-1}=\left(\operatorname{int} T+\gamma_{1}\right) \cup \bigcup_{\gamma \in \Gamma}\left((T+\gamma) \cap\left(T+\gamma_{1}\right)\right)
$$

is a Borel set.

For any $x \in \mathbf{R}^{d}$ we will call $[x]$ the integer part of $x$ and $(x)=x-[x]$ the fractional part of $x$. By Proposition $1,[Z]$ is a random variable and therefore so is $(Z)=Z-[Z]$. Notice that $(Z)$ takes values in the tile $T$.

A point $t \in \mathbf{R}^{d}$ is in $T$ if and only if

$$
t=\sum_{j=1}^{\infty} M^{-j} \gamma_{j}
$$

where for all $j, \gamma_{j} \in \Gamma_{0}$ [5]. Based on the expansion (2.3), define functions $\xi_{j}: \Omega \rightarrow$ $\Gamma_{0}, j=1,2, \ldots$, by

$$
\left(Z_{0}\right)=\sum_{j=1}^{\infty} M^{-j} \xi_{j}
$$

that is, $\xi_{j}(\omega)$ is the element of $\Gamma_{0}$ which appears in the $j$ th term of the tile expansion of $\left(Z_{0}\right)(\omega)$. If there is more than one expansion for a tile point, simply choose one of them.

Proposition 2. Assume that $P\left(\left(Z_{0}\right) \in \partial T\right)=0$. Then $\left\{\xi_{j}\right\}_{j=1}^{\infty}$ is a sequence of random variables and for each $k$

$$
\left(Z_{k}\right)=\sum_{j=1}^{\infty} M^{-j} \xi_{j+k} \quad \text { a.s. }
$$

Proof. From the dilation equation (1.2) and from the decomposition of $Z_{0}$ into its fractional and integer parts, we obtain

$$
M\left[Z_{0}\right]+M\left(Z_{0}\right)=M Z_{0}=G_{1}+Z_{1}=G_{1}+\left[Z_{1}\right]+\left(Z_{1}\right) .
$$

Using (2.4) it follows that

$$
M\left[Z_{0}\right]+\xi_{1}+\sum_{j=1}^{\infty} M^{-j} \xi_{j+1}=G_{1}+\left[Z_{1}\right]+\left(Z_{1}\right) .
$$

The definition of a lattice tiling implies $(\gamma+T) \cap\left(\gamma^{\prime}+\right.$ int $\left.T\right)=\emptyset$ if and only if $\gamma \neq \gamma^{\prime}$. So, if $\left(Z_{1}\right) \in \operatorname{int} T$, then by (2.5), we have

$$
M\left[Z_{0}\right]+\xi_{1}=G_{1}+\left[Z_{1}\right] \text { and }\left(Z_{1}\right)=\sum_{j=1}^{\infty} M^{-j} \xi_{j+1} .
$$

Since $P\left(\left(Z_{0}\right) \in \partial T\right)=0$ and since $Z_{1} \stackrel{d}{=} Z_{0}$, it follows that $P\left(\left(Z_{1}\right) \in \operatorname{int} T\right)=1$, and therefore

$$
\left(Z_{1}\right)=\sum_{j=1}^{\infty} M^{-j} \xi_{j+1} \text { a.s. }
$$

By (2.6) $\xi_{1}=G_{1}+\left[Z_{1}\right]-M\left[Z_{0}\right]$ almost surely and so $\xi_{1}$ is a random variable.

The proof is completed by induction on $k$. 
Define $h: \Gamma \rightarrow \Gamma_{0}$ to be the map which assigns to each element of $\Gamma$ its coset representative.

Proposition 3. Suppose $P\left(\left(Z_{0}\right) \in \partial T\right)=0$. Then for

$$
k=1,2, \ldots, \quad \xi_{k}=h\left(\left[Z_{k}\right]+G_{k}\right) \text { a.s. }
$$

Proof. $P\left(\left(Z_{0}\right) \in \partial T\right)=0$ implies that (2.6) holds. So $\xi_{1}=h\left(G_{1}+\left[Z_{1}\right]\right)$ since coset representatives are unique.

Proposition 2 the fact that $Z_{k}=\left[Z_{k}\right]+\left(Z_{k}\right)$, and the dilation equation (1.2) together lead to

$$
M\left[Z_{k}\right]+\xi_{k+1}+\left(Z_{k+1}\right)=G_{k+1}+\left[Z_{k+1}\right]+\left(Z_{k+1}\right) \text { a.s. }
$$

This implies that $M\left[Z_{k}\right]+\xi_{k+1}=G_{k+1}+\left[Z_{k+1}\right]$ a.s. since $P\left(\left(Z_{k+1}\right) \in \partial T\right)=0$. The uniqueness of coset representatives ensures $\xi_{k+1}=h\left(G_{k+1}+\left[Z_{k+1}\right]\right)$ a.s.

Define $g:\left(\mathbf{R}^{d}\right)^{\infty} \rightarrow \mathbf{R}^{d}$ by

$$
g\left(x_{1}, x_{2}, \ldots\right)=x_{1}+\left[\sum_{j=1}^{\infty} M^{-j} x_{j+1}\right] .
$$

The measurability of $g$ follows from Proposition 1 and from the fact that the projection map is a measurable function.

Proposition 4. Let $Y_{k}:=(h \circ g)\left(G_{k}, G_{k+1}, \ldots\right)$. Then $Y_{1}, Y_{2}, \ldots$ is a stationary and ergodic sequence of random variables.

Proof. The proof follows from the fact that $h \circ g$ is measurable and $\left\{G_{k}\right\}_{k=1}^{\infty}$ is i.i.d.

Corollary 1. If $P\left(\left(Z_{0}\right) \in \partial T\right)=0$, the sequence $\xi_{1}, \xi_{2}, \ldots$ is stationary and ergodic.

Proof. If $P\left(\left(Z_{0}\right) \in \partial T\right)=0$, Proposition 4 implies $\xi_{k}=Y_{k}$ a.s .

\section{NeCESSARY AND SUfFicient CONDITIONS For ABSOLUte CONTINUITy of $Z$}

Throughout this section let $\lambda_{T}:=\frac{\lambda}{\lambda(T)}$ denote Lebesgue measure normalized by the measure of the tile $T$ (if $\Gamma=\mathbf{Z}^{d}$, then $\lambda(T)=1$ )).

Theorem 1. Let $M, \Gamma, \Gamma_{0}$ and random variables $G, Z$ and $\xi_{k}$ be as defined in the previous sections. Suppose $G$ has values in a finite set $\Gamma_{1}$ such that $\Gamma_{0} \subseteq \Gamma_{1} \subset \Gamma$. Then the following are equivalent:

1) The law of $(Z)$ is $\lambda_{T}$ on $T$;

2) The $\xi_{k}$ are independent and uniformly distributed on $\Gamma_{0}$;

3) The law of $Z$ is absolutely continuous with respect to $\lambda$.

Proof. $(1 \Rightarrow 3)$ Since $G$ is bounded, so is $Z$, and therefore $[Z]$ takes on only finitely many values. Let $\Gamma_{2}$ be the range of $[Z]$. One solution of equation (1.2) is $Z \stackrel{d}{=}$ $\sum_{k=1}^{\infty} M^{-k} G_{k}$. Jessen and Wintner's theorem [8] implies that the law of $Z$ must be either purely discrete, purely singular, or purely absolutely continuous. We will rule out the discrete and singular cases. 
First, suppose $Z$ is purely discrete. Then $P(Z=z)>0$ for some $z$. Now,

$$
\begin{aligned}
0 & <P(Z=z)=P([Z]+(Z)=z) \\
& =\sum_{\gamma \in \Gamma_{2}} P([Z]+(Z)=z \mid[Z]=\gamma) P([Z]=\gamma)
\end{aligned}
$$

implies that there exists a $\gamma \in \Gamma_{1}$ such that

$$
P((Z)=z-\gamma \mid[Z]=\gamma) P([Z]=\gamma)>0,
$$

contradicting the assumption that $(Z)$ is uniform.

Second, suppose $Z$ is purely singular with respect to Lebesgue measure. Then there exists $B$ such that $P(Z \in B)=1$ and $\lambda_{T}(B)=0$. So

$$
P([Z]+(Z) \in B)=\sum_{\gamma \in \Gamma_{2}} P([Z]+(Z) \in B \mid[Z]=\gamma) P([Z]=\gamma)=1,
$$

which implies that there exists a $\gamma \in \Gamma_{2}$ such that

$$
P((Z) \in B-\gamma \mid[Z]=\gamma) P([Z]=\gamma) \geq \frac{1}{\left|\Gamma_{2}\right|} .
$$

But under the assumption that $(Z)$ is uniform, $P((Z) \in B-\gamma)=\lambda_{T}(B-\gamma)=$ $\lambda_{T}(B)=0$, a contradiction.

Next, 2$) \Rightarrow 1)$. This proof will be broken into three main steps:

(i) assumption 2) implies $P\left(\left(Z_{0}\right) \in \partial T\right)=0$;

(ii) $\nu:=£(Z)$ and $\lambda_{T}$ agree on sets of the type $M^{-k} T+M^{-k} \gamma, \gamma \in \Gamma$;

(iii) $\nu$ and $\lambda_{T}$ agree on all closed balls.

Remark. The first step is trivial in one dimension. For example, if $M=2, \Gamma=\mathbf{Z}$ and $\Gamma_{0}=\{0,1\}$, then $T=[0,1]$ and

$$
P\left(\sum_{k=1}^{\infty} 2^{-k} \xi_{k} \in \partial T\right)=P\left(\xi_{k}=0 \text { for all } k \text { or } \xi_{k}=1 \text { for all } k\right)=0 .
$$

i) For each $n=0,1,2, \ldots$ let

$$
W_{n}=\sum_{k=1}^{\infty} M^{-k} \xi_{k+n}
$$

Notice that the range of $W_{n}$ is in $T$ and since the sequence $\left\{\xi_{k}\right\}_{k=1}^{\infty}$ is i.i.d., $W_{n} \stackrel{d}{=}$ $W_{0}, n=1,2, \ldots$

Claim. $P\left(W_{0} \in\right.$ int $\left.T\right)>0$.

Proof. Since $\operatorname{int} T \neq \emptyset$ [10], let $B(x ; r) \subset$ int $T$ be an open ball centered at $x$ with radius $r$. Then $x=\sum_{i=1}^{\infty} M^{-i} \gamma_{i}(x)$, where $\gamma_{i}(x) \in \Gamma_{0}$ for all $i$ [5]. Choose $k$ large enough so that

$$
\sum_{i=k}^{\infty}\left\|M^{-i}\right\| \max \left\{\|\gamma\| \mid \gamma \in \Gamma_{0}\right\}<\frac{r}{2}
$$

Let $y=\sum_{i=1}^{k-1} M^{-i} \gamma_{i}(x)$. Note that $y \in B\left(x ; \frac{r}{2}\right)$. Let

$$
S=\left\{t \in T \mid \gamma_{i}(t)=\gamma_{i}(x) \text { for } i=1,2, \ldots, k-1\right\}
$$


Then $S \subseteq B(x ; r)$ and

$$
P\left(W_{0} \in S\right)=P\left(\xi_{1}=\gamma_{1}(x), \ldots, \xi_{k-1}=\gamma_{k-1}(x)\right)=\frac{1}{m^{k-1}} .
$$

So $P\left(W_{0} \in S\right)>0$, which together with $S \subset$ int $T$ implies $P\left(W_{0} \in \operatorname{int} T\right)>0$.

One property of a tiling is that distinct tiles may only intersect on their boundaries. If we set $\Gamma_{\partial}=\{\gamma \in \Gamma \backslash\{0\} \mid T \cap(T+\gamma) \neq \emptyset\}$, then

$$
\partial T=\bigcup_{\gamma \in \Gamma_{\partial}}(T \cap(T+\gamma)) .
$$

Claim. $\left\{W_{n} \in \partial T\right\} \subseteq\left\{W_{n+1} \in \partial T\right\}$ for $n=0,1,2, \ldots$

Proof. Suppose $\omega \in\left\{W_{0} \in \partial T\right\}$; that is,

$$
\sum_{k=1}^{\infty} M^{-k} \xi_{k}(\omega) \in \partial T .
$$

Applying $M$ to both sides and using properties of tiles yields

$$
W_{1}(\omega)=\sum_{k=1}^{\infty} M^{-k} \xi_{k+1}(\omega) \in \partial M T-\xi_{1}(\omega) .
$$

Set $\gamma_{1}:=\xi_{1}(\omega)$. By the self-affine property of the tiling, $\partial M T \subseteq \bigcup_{\gamma \in \Gamma_{0}}(\gamma+\partial T)$. Therefore, (3.2) becomes

$$
W_{1}(\omega) \in \bigcup_{\gamma \in \Gamma_{0}}\left(\left(\gamma-\gamma_{1}\right)+\partial T\right),
$$

implying that for at least one $\gamma \in \Gamma_{0}, W_{1}(\omega) \in\left(\gamma-\gamma_{1}\right)+\partial T$. So

$$
W_{1}(\omega) \in\left(\left(\gamma-\gamma_{1}\right)+\partial T\right) \cap T \text {. }
$$

If $\gamma=\gamma_{1}$, then $W_{1}(\omega) \in \partial T$; if $\gamma \neq \gamma_{1}$, then int $T \cap\left(\gamma-\gamma_{1}+\right.$ int $\left.T\right)=\emptyset$, so $W_{1}(\omega) \in \partial T$. We have shown that $\left\{W_{0} \in \partial T\right\} \subseteq\left\{W_{1} \in \partial T\right\}$. By the same argument, $\left\{W_{n} \in \partial T\right\} \subseteq\left\{W_{n+1} \in \partial T\right\}$ for each $n$.

Claim. $\mathrm{P}\left(W_{0} \in \partial T\right)=0$.

Suppose not. Set $B_{k}=\left\{W_{k} \in \partial T\right\}$ and $B=\bigcup_{k=0}^{\infty} B_{k}$. Notice that since the $B_{k}$ are nested, $B \in \bigcap_{n=1}^{\infty} \sigma\left(\xi_{n}, \xi_{n+1}, \ldots\right)$. By the Kolmogorov 0-1 law for independent random variables $P(B)=1$, because $\left\{W_{0} \in \partial T\right\} \subset B$ and $P\left(W_{0} \in \partial T\right)>0$. Furthermore,

$$
1=P(B)=\lim _{k \rightarrow \infty} P\left(W_{k} \in \partial T\right)=P\left(W_{0} \in \partial T\right),
$$

with the last equality following from the fact that the sequence $\xi_{1}, \xi_{2}, \ldots$ is i.i.d. But this is a contradiction of the fact that $P\left(W_{0} \in \operatorname{int} T\right)>0$. So $P\left(W_{0} \in \partial T\right)=0$.

Since $W_{0}=\left(Z_{0}\right)$ almost surely we have shown that $P\left(\left(Z_{0}\right) \in \partial T\right)=0$, concluding the first step.

(ii) To begin the second step of the proof, fix $\gamma \in \Gamma$ and $k \in \mathbf{N}$. Then

$$
\lambda\left(M^{-k} T+M^{-k} \gamma\right)=\lambda\left(M^{-k} T\right)=\frac{\lambda(T)}{m^{k}} .
$$


By Proposition 2 and (i) $\left(Z_{k}\right)=\sum_{i=1}^{\infty} M^{-i} \xi_{i+k}$ a.s. Now,

$$
\begin{aligned}
P\left(\left(Z_{0}\right) \in M^{-k} T+M^{-k} \gamma\right) & =P\left(M^{k}\left(Z_{0}\right) \in T+\gamma\right) \\
& =P\left(M^{k} \sum_{i=1}^{\infty} M^{-i} \xi_{i} \in T+\gamma\right) \\
& =P\left(\sum_{j=1-k}^{0} M^{-j} \xi_{j+k}+\sum_{j=1}^{\infty} M^{-j} \xi_{j+k} \in T+\gamma\right) \\
& =P\left(L(k)+\left(Z_{k}\right) \in T+\gamma\right),
\end{aligned}
$$

where $L(k):=\sum_{j=1-k}^{0} M^{-j} \xi_{j+k}$. Notice that $L(k)$ is a function of finitely many $\xi_{i}$ and has values in the lattice; therefore,

$$
\begin{aligned}
P\left(L(k)+\left(Z_{k}\right) \in T+\gamma\right) & =\sum_{\gamma^{\prime}} P\left(\left(Z_{k}\right) \in T+\gamma-\gamma^{\prime}, L(k)=\gamma^{\prime}\right) \\
& =P\left(\left(Z_{k}\right) \in T, L(k)=\gamma\right) .
\end{aligned}
$$

The last equality follows since all the terms in the sum are zero except when $\gamma^{\prime}=\gamma$ as a consequence of $P\left(\left(Z_{k}\right) \in \partial T\right)=0$. Furthermore,

$$
\begin{aligned}
P\left(\left(Z_{k}\right) \in T, L(k)=\gamma\right) & =P(L(k)=\gamma) \\
& =P\left(M^{k-1} \xi_{1}+\cdots+M \xi_{k-1}+\xi_{k}=\gamma\right) \\
& =P\left(M\left(M^{k-2} \xi_{1}+\cdots+\xi_{k-1}\right)+\xi_{k}=\gamma\right) .
\end{aligned}
$$

Since each $\gamma \in \Gamma$ has a unique representation $\gamma=\gamma_{0}+M \gamma^{\prime \prime}$, (3.3) becomes

$$
\begin{aligned}
P\left(\xi_{k}\right. & \left.=\gamma_{0}, M^{k-2} \xi_{1}+\cdots+\xi_{k-1}=\gamma^{\prime \prime}\right) \\
& =P\left(\xi_{k}=\gamma_{0}, \xi_{k-1}=\gamma_{1}, M^{k-3} \xi_{1}+\cdots+\xi_{k-2}=\gamma^{\prime \prime \prime}\right) \\
& =P\left(\xi_{k}=\gamma_{0}, \xi_{k-1}=\gamma_{1}, \ldots, \xi_{1}=\gamma_{k-1}\right) \\
& =\prod_{i=1}^{k} P\left(\xi_{i}=\gamma_{k-i}\right)=\frac{1}{m^{k}} .
\end{aligned}
$$

So $£((Z))$ and $\frac{\lambda}{\lambda(T)}$ are equal on sets of the type $M^{-k} T+M^{-k} \gamma, \gamma \in \Gamma$ and $k \in N$.

(iii) We now show that $£((Z))$ and $\lambda_{T}$ agree on all closed balls in $\mathbf{R}^{d}$.

Set $\nu:=£((Z))$, and suppose there is a closed ball $B(x, r)$ on which the measures do not agree. Assume first that $\nu(B(x, r) \cap T)>\lambda_{T}(B(x, r) \cap T)$. There exists $\eta>0$, such that $\nu(B(x, r) \cap T)>\lambda_{T}(B(x, r+\eta) \cap T)$. Choose $k_{0}$ such that $\operatorname{diam}\left(M^{-k_{0}} T\right)<\frac{\eta}{2}$. Set

$$
D=\bigcup\left\{M^{-k_{0}} T+M^{-k_{0}} \gamma \mid \gamma \in M^{k_{0}} B\left(x, r+\frac{\eta}{2}\right)\right\} .
$$

Claim. $B(x, r) \subseteq D \subseteq B(x, r+\eta)$.

Proof. Let $y \in B(x, r)$. Since $\mathbf{R}^{d}=\bigcup_{\gamma \in \Gamma}\left(M^{-k_{0}} T+M^{-k_{0}} \gamma\right)$, there is a $\gamma \in \Gamma$ such that $y \in M^{-k_{0}} T+M^{-k_{0}} \gamma$. So $y=z+M^{-k_{0}} \gamma$, for some $z \in M^{-k_{0}} T$. If $z \in M^{-k_{0}} T$, then $\|z\| \leq \operatorname{diam}\left(M^{-k_{0}} T\right)$ since $0 \in T$. Now

$$
\left\|M^{-k_{0}} \gamma-x\right\| \leq\|y-x\|+\|z\| \leq r+\operatorname{diam}\left(M^{-k_{0}} T\right) \leq r+\frac{\eta}{2},
$$


that is,

$$
M^{-k_{0}} \gamma \in B\left(x, r+\frac{\eta}{2}\right)
$$

which means $y \in D$.

Now suppose that $y \in D$. Then $y=z+M^{-k_{0}} \gamma$ for some $z \in M^{-k_{0}} T$ and $\gamma \in B\left(x, r+\frac{\eta}{2}\right)$, and

$$
\|y-x\| \leq\left\|M^{-k_{0}} \gamma-x\right\|+\|z\| \leq r+\eta
$$

so $y \in B(x, r+\eta)$. This completes the proof of the claim.

Thus $\lambda_{T}(B(x, r+\eta) \cap T) \geq \lambda_{T}(D \cap T)$ and $\nu(D \cap T) \geq \nu(B(x, r) \cap T)$. If we can show that $\lambda_{T}(D \cap T)=\nu(D \cap T)$, we will obtain a contradiction. To see this, recall that by the self-affine property of the tiling, we can write

$$
T=\bigcup_{\gamma \in \Gamma_{k_{0}}} M^{-k_{0}} T+M^{-k_{0}} \gamma
$$

where $\Gamma_{k_{0}}=\Gamma_{0}+M \Gamma_{0}+\cdots+M^{k_{0}-1} \Gamma_{0}$. If $\gamma \in \Gamma_{k_{0}}$, then $M^{-k_{0}} T+M^{-k_{0}} \gamma \subset T$, so $\operatorname{int}\left(M^{-k_{0}} T+M^{-k_{0}} \gamma\right) \subset T$. If $\gamma \notin \Gamma_{k_{0}}$, then $T \cap \operatorname{int}\left(M^{-k_{0}} T+M^{-k_{0}} \gamma\right)=\emptyset$. If not, there is an $x$ in $T \cap \operatorname{int}\left(M^{-k_{0}} T+M^{-k_{0}} \gamma\right)$. Since $x \in T, x$ is in one of the sets in the right-hand side of (3.4); that is, $x \in M^{-k_{0}} T+M^{-k_{0}} \gamma^{\prime}$, where $\gamma^{\prime} \in \Gamma_{k_{0}}$. So

$$
x \in\left(M^{-k_{0}} T+M^{-k_{0}} \gamma^{\prime}\right) \cap \operatorname{int}\left(M^{-k_{0}} T+M^{-k_{0}} \gamma\right),
$$

which implies $M^{k_{0}} x \in\left(T+\gamma^{\prime}\right) \cap \operatorname{int}(T+\gamma)$. This contradicts the fact that distinct translates of $T$ are disjoint except at the boundary. So,

$$
\text { either } \operatorname{int}\left(M^{-k_{0}} T+M^{-k_{0}} \gamma\right) \subset T \text { or } \operatorname{int}\left(M^{-k_{0}} T+M^{-k_{0}} \gamma\right) \subset T^{c} \text {. }
$$

Set $C=\Gamma_{k_{0}} \cap M^{k_{0}} B\left(x, r+\frac{\eta}{2}\right)$ and $C^{\prime}=\left(\Gamma \backslash \Gamma_{k_{0}}\right) \cap M^{k_{0}} B\left(x, r+\frac{\eta}{2}\right)$. Then

$$
D \cap T=\left(\left(\bigcup_{\gamma \in C} M^{-k_{0}} T+M^{-k_{0}} \gamma\right) \cap T\right) \cup\left(\left(\bigcup_{\gamma \in C^{\prime}} M^{-k_{0}} T+M^{-k_{0}} \gamma\right) \cap T\right) .
$$

The second intersection consists only of boundary points of $T$. Since $\nu(\partial T)=0$, then

$$
\nu(D \cap T)=\nu\left(\left(\bigcup_{\gamma \in C} M^{-k_{0}} T+M^{-k_{0}} \gamma\right) \cap T\right)
$$


and $\nu\left(\partial\left(M^{-k_{0}} T+M^{-k_{0}} \gamma\right)\right)=0$. The Lebesgue measure of $\partial T$ is zero [10], so $\lambda\left(\partial M^{-k_{0}} T\right)=0$. Thus we have

$$
\begin{aligned}
\lambda_{T}(D \cap T) & =\lambda_{T}\left(\bigcup_{\gamma \in \Gamma_{k_{0}}} M^{-k_{0}} T+M^{-k_{0}} \gamma\right) \\
& =\sum_{\gamma \in \Gamma_{k_{0}}} \lambda_{T}\left(M^{-k_{0}} T+M^{-k_{0}} \gamma\right) \\
& =\sum_{\gamma \in \Gamma_{k_{0}}} \nu\left(M^{-k_{0}} T+M^{-k_{0}} \gamma\right) \\
& =\nu\left(\bigcup_{\gamma \in \Gamma_{k_{0}}} M^{-k_{0}} T+M^{-k_{0}} \gamma\right)=\nu(D \cap T)
\end{aligned}
$$

As mentioned above, the fact that $\lambda_{T}(D \cap T)=\nu(D \cap T)$ implies

$$
\lambda_{T}(B(x, r+\eta) \cap T) \geq \nu(B(x, r) \cap T)
$$

which contradicts $\nu(B(x, r) \cap T)>\lambda_{T}(B(x, r) \cap T)$. So we conclude that $\lambda_{T} \leq \nu$ on all closed balls. Repeating the proof with the roles of $\nu$ and $\lambda_{T}$ reversed yields that $\nu$ and $\lambda_{T}$ agree on all closed balls.

Hoffmann-Jørgensen proved that Radon probabilities which agree on all closed balls in $\mathbf{R}^{d}$ agree on all Borel sets. (Corollary 5 in 7 ), which completes the proof that 2$) \Rightarrow 1$ ).

In order to prove 3$) \Rightarrow 2$ ), we need a version of the Kakutani Dichotomy for stationary ergodic sequences.

Lemma 1. Let $\left\{\xi_{k}^{\prime}\right\}_{k=1}^{\infty}$ be a stationary, ergodic sequence, such that each $\xi_{k}^{\prime}$ is uniform with values in $\Gamma_{0}$. Let $\left\{\xi_{k}\right\}_{k=1}^{\infty}$ be a stationary, ergodic sequence, such that each $\xi_{k}$ has values in $\Gamma_{0}$, but is not uniform. Then $\mu=£\left(\xi_{1}, \xi_{2}, \ldots\right)$ and $\mu^{\prime}=£\left(\xi_{1}^{\prime}, \xi_{2}^{\prime}, \ldots\right)$ are mutually singular.

Proof. Let $\mu=\mu_{a}+\mu_{s}$, where $\mu_{a}<<\mu^{\prime}$ and $\mu_{s} \perp \mu^{\prime}$. Suppose $\mu_{a}(\Omega)>0$.

Since $\mu \neq \mu^{\prime}$, there must be a cylindrical set $A$ such that $\mu_{a}(A) \neq \mu^{\prime}(A)$. (If not, then $\mu_{a}=\mu^{\prime}$, which implies $\mu=\mu^{\prime}$, contradicting the assumption that $\mu \neq \mu^{\prime}$.) Let $f=1_{A}$, then we get

$$
\begin{aligned}
\int_{\Omega} f\left(x_{1}, . ., x_{n}\right) d \mu_{a}(x) & \neq \int_{\Omega} f\left(x_{1}, . ., x_{n}\right) d \mu^{\prime}(x), \\
E_{\mu_{a}}(f) & \neq E_{\mu^{\prime}}(f) .
\end{aligned}
$$

Set $c=E_{\mu_{a}}(f)$ and $c^{\prime}=E_{\mu^{\prime}}(f)$. The fact that $\left\{\xi_{k}\right\}_{k=1}^{\infty}$ and $\left\{\xi_{k}^{\prime}\right\}_{k=1}^{\infty}$ are ergodic sequences means that the shift operator is an ergodic operator for $(\Omega, S, \mu)$ and $\left(\Omega, S, \mu^{\prime}\right)$ respectively, where $\Omega=\Gamma_{0}^{\infty}$. Applying the Ergodic Theorem (with $f$ ) and the fact that the sequences are stationary, it follows that

1) $\quad \frac{1}{k} \sum_{i=0}^{k-1} f\left(x_{1+i}, \ldots, x_{n+i}\right) \stackrel{k \rightarrow \infty}{\rightarrow} c$ a.s. $\mu_{a}$,
2) $\quad \frac{1}{k} \sum_{i=0}^{k-1} f\left(x_{1+i}, \ldots, x_{n+i}\right) \stackrel{k \rightarrow \infty}{\rightarrow} c^{\prime}$ a.s. $\mu^{\prime}$.

So, 1) is true for all $\left\{x_{i}\right\}_{i=1}^{\infty} \in \Omega \backslash N$, where $\mu_{a}(N)=0$ and 2) is true for all $\left\{x_{i}\right\}_{i=1}^{\infty} \in \Omega \backslash N^{\prime}$, where $\mu^{\prime}\left(N^{\prime}\right)=0$. 
Define $M:=N \cup N^{\prime}$. Notice that $\mu_{a}(M) \leq \mu_{a}(N)+\mu_{a}\left(N^{\prime}\right)=\mu_{a}\left(N^{\prime}\right)$. Since $\mu_{a}<<\mu^{\prime}$ and $\mu^{\prime}\left(N^{\prime}\right)=0$, we have $\mu_{a}\left(N^{\prime}\right)=0$ and so $\mu_{a}(M)=0$. We have assumed that $\mu_{a}(\Omega)>0$; therefore, $\mu_{a}(M)=0$ implies that $\mu_{a}(\Omega \backslash M)>0$; that is, $\mu_{a}\left((\Omega \backslash N) \cap\left(\Omega \backslash N^{\prime}\right)\right)>0$, which means that there is a sequence $\left\{x_{i}\right\}_{i=1}^{\infty} \in$ $(\Omega \backslash N) \cap\left(\Omega \backslash N^{\prime}\right)$ such that

$$
\frac{1}{k} \sum_{i=0}^{k-1} f\left(x_{1+i}, \ldots, x_{n+i}\right) \stackrel{k \rightarrow \infty}{\rightarrow} c \text { and } \frac{1}{k} \sum_{i=1}^{k-1} f\left(x_{1+i}, \ldots, x_{n+i}\right) \stackrel{k \rightarrow \infty}{\rightarrow} c^{\prime} .
$$

This is a contradiction, since $c \neq c^{\prime}$. Therefore, $\mu_{a}=0$ and thus, $\mu \perp \mu^{\prime}$.

Now we are ready to show that 3$) \Rightarrow 2$ ).

First, we note that $£(Z)<<\lambda_{T}$ implies that $£((Z))<<\lambda_{T}$. To see this, observe that for $E \in \mathcal{B}\left(\mathbf{R}^{d}\right)$,

$$
\begin{aligned}
P((Z) \in E) & =P(Z-[Z] \in E)=\sum_{\gamma \in \Gamma} P(Z \in E+\gamma,[Z]=\gamma) \\
& \leq \sum_{\gamma \in \Gamma} P(Z \in E+\gamma) .
\end{aligned}
$$

If $\lambda_{T}(E)=0$, then $\lambda_{T}(E+\gamma)=0$ and so $P(Z \in E+\gamma)=0$ for all $\gamma \in \Gamma$ by the assumption of absolute continuity of $£(Z)$. Then (3.5) implies $P((Z) \in E)=0$. So $£((Z))<<\lambda_{T}$.

Since $\lambda(\partial T)=0, £((Z))<<\lambda_{T}$ implies that $P((Z) \in \partial T)=0$. Therefore, if we define $s: \Gamma_{0}^{\infty} \rightarrow \mathbf{R}$ by

$$
s\left(x_{1}, x_{2}, \ldots\right):=\sum_{i=1}^{\infty} M^{-i} x_{i},
$$

If $\Gamma_{0}^{\infty}$ is equipped with the product topology, $s$ is continuous. By Proposition 2 for every Borel set $F$ the following holds true:

$$
\begin{aligned}
£((Z))(F) & =£\left(\sum_{i=1}^{\infty} M^{-i} \xi_{i}\right)(F)=P\left(s\left(\xi_{1}, \xi_{2}, \ldots\right) \in F\right) \\
& =P\left(\left(\xi_{1}, \xi_{2}, \ldots\right) \in s^{-1}(F)\right)=\mu\left(s^{-1}(F)\right),
\end{aligned}
$$

where $\mu=£\left(\xi_{1}, \xi_{2}, \ldots\right)$. Corollary 1 assures that the sequence $\left\{\xi_{k}\right\}_{k=1}^{\infty}$ is stationary and ergodic. Let $\mu^{\prime}=£\left(\xi_{1}^{\prime}, \xi_{2}^{\prime}, \ldots\right)$, where $\left\{\xi_{k}^{\prime}\right\}_{k=1}^{\infty}$ is an i.i.d. sequence with $\xi_{1}^{\prime}$ uniform on $\Gamma_{0}$. Suppose that $\mu \neq \mu^{\prime}$. Then by Lemma 1, $\mu \perp \mu^{\prime}$. So there is a set $B \subset \mathfrak{B}\left(\Gamma_{0}^{\infty}\right)$ such that $\mu(B)=1$ and $\mu^{\prime}(B)=0$. Set $A=s(B)$. Since $\Gamma_{0}^{\infty}$ is a Polish space and $s$ is continuous, $A$ being the continuous image of a Borel set, is an analytic set. As such, $A$ is universally measurable [13]. Let $C$ and $D$ be Borel sets so that $C \subseteq A \subseteq D$ and $\lambda(C)=\lambda(A)=\lambda(D)$. Since the Lebesgue measure of boundary of a tile is 0 , we may assume that $C$ does not contain any points on the boundary of tiles (the union of the tiles boundaries is a Borel set). This implies that $s^{-1}(C) \subseteq B$. From the proof of 2$) \Rightarrow 1$ ) it follows that $£\left(s\left(\xi_{1}^{\prime}, \xi_{2}^{\prime}, \ldots\right)\right)=\lambda_{T}$. Now

$$
\begin{aligned}
0 & =\mu^{\prime}(B)=P\left(\left(\xi_{1}^{\prime}, \xi_{2}^{\prime}, \ldots\right) \in B\right) \geq P\left(\left(\xi_{1}^{\prime}, \xi_{2}^{\prime}, \ldots\right) \in s^{-1}(C)\right) \\
& =P\left(s\left(\xi_{1}^{\prime}, \xi_{2}^{\prime}, \ldots\right) \in C\right)=\lambda_{T}(C)=\lambda_{T}(A)
\end{aligned}
$$


We also have that

$$
1=\mu(B)=P\left(\left(\xi_{1}, \xi_{2}, \ldots\right) \in B\right) \leq P\left(s\left(\xi_{1}, \xi_{2}, \ldots\right) \in D\right)=£((Z))(A)
$$

where the last equality follows from (3.6). This contradicts the fact that $£((Z))<<$ $\lambda_{T}$. Therefore $\mu=\mu^{\prime}$, i.e. $\xi_{i}, i=1,2, \ldots$, are i.i.d. and $\xi_{1}$ is uniform on $\Gamma_{0}$. This completes the proof of 3$) \Rightarrow 2$ ) and thus of Theorem 1 .

\section{Conditions For independence of $\left\{\xi_{k}\right\}$}

In Theorem 11 the existence of a density of the solution $Z$ to $(1.2)$ is equivalent to the fact that the stationary, ergodic sequence $\left\{\xi_{k}\right\}_{k=1}^{\infty}$ is a sequence of independent random variables and that $\xi_{1}$ is uniform on $\Gamma_{0}$. In this section we first investigate the effects of uniformity of $\xi_{1}$ on the distributions of $G_{1}$ and $\left[Z_{1}\right]$; the results are then summarized in Theorem 2] In Theorem 3, we give a sufficient condition on $G_{1}$ for the independence and uniformity of the sequence $\left\{\xi_{k}\right\}_{k=1}^{\infty}$.

By Proposition 3, $\xi_{k}=h\left(G_{k}+\left[Z_{k}\right]\right)$ a.s., provided that $P\left(\left(Z_{0}\right) \in \partial T\right)=0$. In order to describe the effects of uniformity of $\xi_{k}$, it suffices to consider the relationship between $G_{1},\left[Z_{1}\right]$ and $\xi_{1}$.

Let $p_{i}=P\left(\left[Z_{1}\right] \cong \gamma_{i}\right)$ and $q_{i}=P\left(G_{1} \cong \gamma_{i}\right)$ for $i=0,1, \ldots, m-1$, where $\gamma \cong \gamma_{i}$ means that the lattice point $\gamma$ is in the coset represented by $\gamma_{i}$. Recalling that $G_{1}$ and $Z_{1}$ are independent we have

$$
\begin{aligned}
P\left(\xi_{1}=\gamma_{k}\right) & =P\left(h\left(G_{1}+\left[Z_{1}\right]\right)=\gamma_{k}\right) \\
& =\sum_{\gamma_{i}+\gamma_{j} \cong \gamma_{k}} P\left(G_{1} \cong \gamma_{i},\left[Z_{1}\right] \cong \gamma_{j}\right) \\
& =\sum_{\gamma_{i}+\gamma_{j} \cong \gamma_{k}} P\left(G_{1} \cong \gamma_{i}\right) P\left(\left[Z_{1}\right] \cong \gamma_{j}\right) \\
& =\sum_{\gamma_{i}+\gamma_{j} \cong \gamma_{k}} q_{i} p_{j} .
\end{aligned}
$$

Due to the uniqueness of equivalence class representatives, there are exactly $m$ terms in the right-hand sides the equation. Now if $p_{i}=\frac{1}{m}$ for all $i$ or $q_{i}=\frac{1}{m}$ for all $\mathrm{i}$ then $\xi_{i}$ are uniform. Assuming that $\xi_{1}$ is uniform on $\Gamma_{0}=\left\{\gamma_{0}, \ldots, \gamma_{m-1}\right\}$ we have,

$$
\begin{aligned}
\frac{1}{m}= & q_{0} p_{0}+q_{1} p_{1}+\cdots+q_{m-1} p_{m-1}, \\
\frac{1}{m}= & q_{m-1} p_{0}+q_{0} p_{1}+\cdots+q_{m-2} p_{m-1}, \\
\frac{1}{m}= & q_{m-2} p_{0}+q_{m-1} p_{1}+\cdots+q_{m-3} p_{m-1}, \\
& \cdots \\
\frac{1}{m}= & q_{1} p_{0}+q_{2} p_{1}+\cdots+q_{0} p_{m-1},
\end{aligned}
$$

or, in matrix form, $Q X=\frac{1}{m}\left[\begin{array}{llll}1 & 1 & \cdots & 1\end{array}\right]^{T}$, where $X=\left[\begin{array}{llll}p_{0} & p_{1} & \cdots & p_{m-1}\end{array}\right]^{T}$. Notice that the rows as well as the columns of $Q$ sum to 1 . Without loss of generality, we may assume that $q_{0} \geq q_{1} \geq \cdots \geq q_{m-1}$; if not, just reindex $\Gamma_{0}$ so that this ordering holds. It is obvious that $p_{i}=\frac{1}{m}, i=0, \ldots, m-1$, is a solution of the system; we will show that it is unique by showing that the eigenvalues of the matrix $Q$ are different from zero. 
Let $\alpha_{k}$ be the $k$ th root of $z^{m}=1$. Direct computation shows that the eigenvalues of $Q$ are $\eta_{k}=\sum_{j=0}^{m-1} q_{j} \alpha_{k}^{j}$ and the associated eigenvectors are

$$
v_{k}=\left[\begin{array}{lllll}
1 & \alpha_{k} & \alpha_{k}^{2} & \cdots & \alpha_{k}^{m-1}
\end{array}\right]^{T},
$$

for $k=0,1, \ldots, m-1$.

Remark 1 . If $k \in\{0,1, \ldots, m-1\}$ and $m$ are relatively prime, then

$$
\left\{e^{\frac{2 \pi i j k}{m}} \mid j=0,1, \ldots, m-1\right\}
$$

is equal to the set of distinct roots of $z^{m}=1$.

Definition 1. We say that the set $\left\{q_{j}\right\}_{j=0}^{m-1}$ has a cycle of length $r$ if

$$
\begin{aligned}
q_{0} & =q_{1}=\cdots=q_{r-1}, \\
q_{r} & =q_{r+1}=\cdots=q_{2 r-1}, \\
& \ldots \\
q_{m-r} & =\cdots=q_{m-1} .
\end{aligned}
$$

The trivial case $r=1$ is excluded.

So, for example, the set $\left\{\frac{1}{4}, \frac{1}{4}, \frac{1}{5}, \frac{1}{5}, \frac{1}{20}, \frac{1}{20}\right\}$ has a cycle of length 2 while the set $\left\{\frac{1}{4}, \frac{1}{4}, \frac{1}{6}, \frac{1}{6}, \frac{1}{6}\right\}$ has no cycle. Note that if $\left\{q_{j}\right\}_{j=0}^{m-1}$ has a cycle of length $r$, then $r$ divides $m$.

Lemma 2. Zero is an eigenvalue of $Q$ if and only if $\left\{q_{j}\right\}_{j=0}^{m-1}$ has a cycle.

Proof. $(\Leftarrow)$ The case of a cycle of length $m$ is trivial.

Now consider a cycle of length $r$, where $r<m$. Denote the greatest common divisor of $m$ and $r$ by $(m, r)$. We claim that $\eta \frac{m}{r}=0$ (recall that $r$ divides $m$ ). Since $\left\{q_{j}\right\}_{j=0}^{m-1}$ has a cycle of length $r$ and $\frac{2 \pi i j \frac{m}{r}}{m}=\frac{2 \pi i j}{r}$, we have

$$
\begin{aligned}
\eta_{\frac{m}{r}} & =\sum_{j=0}^{r-1} q_{j} e^{\frac{2 \pi i j}{r}}+\sum_{j=r}^{2 r-1} q_{j} e^{\frac{2 \pi i j}{r}}+\cdots+\sum_{j=(m, r)-1}^{m-1} q_{j} e^{\frac{2 \pi i j}{r}} \\
& =q_{0} \sum_{j=0}^{r-1} e^{\frac{2 \pi i j}{r}}+q_{r} \sum_{j=r}^{2 r-1} e^{\frac{2 \pi i j}{r}}+\cdots+q_{(m, r)-1} \sum_{j=(m, r)-1}^{m-1} e^{\frac{2 \pi i j}{r}} \\
& =0 .
\end{aligned}
$$

$(\Rightarrow)$ The assumption that there is no cycle implies $q_{0}>q_{m-1}$. First we will show that $\eta_{k} \neq 0$ in the case that $(k, m)=1$. Define $l_{0}:=\max \left\{k: q_{k}=q_{0}\right\}$ and inductively $l_{i+1}:=\max \left\{k: q_{k}=q_{l_{i}+1}\right\}, i=0,1,2, \ldots, n-2$, that is, there are $n$ different values in the set of $q$ 's. Notice that $l_{n-1}=m-1$ and $q_{l_{0}}=q_{0}$. Now,

$$
\begin{aligned}
\eta_{k}(1- & \left.e^{\frac{2 \pi i k}{m}}\right) \\
= & q_{0}+\left(q_{1}-q_{0}\right) e^{\frac{2 \pi i k}{m}}+\cdots+\left(q_{m-1}-q_{m-2}\right) e^{\frac{2 \pi i k(m-1)}{m}}-q_{m-1} e^{\frac{2 \pi i k m}{m}} \\
= & \left(q_{0}-q_{m-1}\right)+\left(q_{1}-q_{0}\right) e^{\frac{2 \pi i k}{m}}+\cdots+\left(q_{m-1}-q_{m-2}\right) e^{\frac{2 \pi i k(m-1)}{m}} \\
= & \left(q_{l_{0}}-q_{l_{n-1}}\right)+\left(q_{l_{0}+1}-q_{l_{0}}\right) e^{\frac{2 \pi i k}{m}\left(l_{0}+1\right)}+\left(q_{l_{1}+1}-q_{l_{1}}\right) e^{\frac{2 \pi i k}{m}\left(l_{1}+1\right)} \\
& +\cdots+\left(q_{l_{n-2}+1}-q_{l_{n-2}}\right) e^{\frac{2 \pi i k}{m}\left(l_{n-2}+1\right)} .
\end{aligned}
$$


If we set

$$
z_{0}=\left(q_{l_{0}}-q_{l_{0}+1}\right) e^{\frac{2 \pi i k}{m}\left(l_{0}+1\right)}+\cdots+\left(q_{l_{n-2}}-q_{l_{n-2}+1}\right) e^{\frac{2 \pi i k}{m}\left(l_{n-2}+1\right)},
$$

we have $\eta_{k}\left(1-e^{\frac{2 \pi i k}{m}}\right)=q_{0}-q_{m-1}-z_{0}$, and

$$
\left|\eta_{k}\left(1-e^{\frac{2 \pi i k}{m}}\right)\right| \geq q_{0}-q_{m-1}-\left|z_{0}\right| .
$$

We claim $\left|z_{0}\right|<q_{0}-q_{m-1}$. Observe

$$
\begin{aligned}
\left|z_{0}\right| \leq & q_{l_{0}}-q_{l_{0}+1} \\
& +\left|\left(q_{l_{1}}-q_{l_{1}+1}\right) e^{\frac{2 \pi i k}{m}\left(l_{1}+1\right)}+\cdots+\left(q_{l_{n-2}}-q_{l_{n-2}+1}\right) e^{\frac{2 \pi i k}{m}\left(l_{n-2}+1\right)}\right| \\
\leq & q_{l_{0}}-q_{l_{n-4}+1}+\left|\left(q_{l_{n-3}}-q_{l_{n-3}+1}\right)+\left(q_{l_{n-2}}-q_{l_{n-2}+1}\right) e^{\frac{2 \pi i k}{m}\left(l_{n-2}-l_{n-3}\right)}\right| .
\end{aligned}
$$

Since $k>0$, and $k$ and $m$ are relatively prime, $e^{\frac{2 \pi i k}{m}\left(l_{n-2}-l_{n-3}\right)}$ has a nonzero imaginary part and so $\left(q_{l_{n-2}}-q_{l_{n-2}+1}\right) e^{\frac{2 \pi i k}{m}\left(l_{n-2}-l_{n-3}\right)}$ cannot be a positive scalar multiple of $q_{l_{n-3}}-q_{l_{n-3}+1}$. Therefore

$$
\begin{aligned}
\left|z_{0}\right| & <q_{l_{0}}-q_{l_{n-4}+1}+q_{l_{n-3}}-q_{l_{n-3}+1}+\left|\left(q_{l_{n-2}}-q_{l_{n-2}+1}\right) e^{\frac{2 \pi i k}{m}\left(l_{n-2}-l_{n-3}\right)}\right| \\
& =q_{0}-q_{m-1} .
\end{aligned}
$$

So $\left|\eta_{k}\left(1-e^{\frac{2 \pi i k}{m}}\right)\right| \geq q_{0}-q_{m-1}-\left|z_{0}\right|>0$, which completes the case $(k, m)=1$.

Suppose now that $(k, m)>1$. Set

$$
m_{1}:=\frac{m}{(k, m)}, k_{1}:=\frac{k}{(k, m)} \text { and } q_{j}^{\prime}=\sum_{i \cong j} q_{i}
$$

where $i \cong j$ means $i=j \bmod m_{1}$. Notice that $q_{0}^{\prime} \geq q_{1}^{\prime} \geq \cdots \geq q_{m_{1}-1}^{\prime}$ and $\left(k_{1}, m_{1}\right)=$ 1. Rewriting $\eta_{k}$ as

$$
\eta_{k}=\sum_{j=0}^{m-1} q_{j} e^{\frac{2 \pi i j k}{m}}=\sum_{j=0}^{m_{1}-1} q_{j}^{\prime} e^{\frac{2 \pi i j k_{1}}{m_{1}}}
$$

we may apply the previous case because the absence of a cycle implies $q_{0}^{\prime}>q_{m_{1}-1}^{\prime}$.

We can summarize the above in the following theorem: (Recall that $p_{i}=$ $P\left(\left[Z_{1}\right] \cong \gamma_{i}\right)$ and $q_{i}=P\left(G_{1} \cong \gamma_{i}\right)$.)

Theorem 2. The random variable $\xi_{1}$ is uniform on $\Gamma_{0}$ if and only if one of the following two statements holds:

(i) if $\left\{q_{j}\right\}_{j=1}^{m-1}$ has no cycles, then $p_{i}=\frac{1}{m}$ for $i=0, \ldots, m-1$, or

(ii) if $\left\{p_{j}\right\}_{j=1}^{m-1}$ has no cycles, then $q_{i}=\frac{1}{m}$ for $i=0, \ldots, m-1$.

The next theorem gives a condition on the distribution of $G_{1}$ which will guarantee the independence of the sequence $\left\{\xi_{k}\right\}_{k=1}^{\infty}$.

Theorem 3. If $P\left(G_{1} \cong \gamma_{i}\right)=\frac{1}{m}$ for $i=1, \ldots, m$, then $\xi_{1}, \xi_{2}, \ldots$ are independent and $\xi_{1}$ is uniform. 
Proof. Uniformity of $\xi_{1}$ follows from Theorem 2, Let $k_{1}<k_{2}<\cdots<k_{n}$. We proceed by induction on $n$.

Suppose $n=2$. Then

$$
\begin{aligned}
P\left(\xi_{k_{1}}=\gamma_{k_{1}}, \xi_{k_{2}}=\gamma_{k_{2}}\right) & =P\left(G_{k_{1}}+\left[Z_{k_{1}}\right] \cong \gamma_{k_{1}}, \xi_{k_{2}}=\gamma_{k_{2}}\right) \\
& =\sum_{i=0}^{m-1} P\left(G_{k_{1}} \cong \gamma_{i},\left[Z_{k_{1}}\right] \cong \gamma_{j(i)}, \xi_{k_{2}}=\gamma_{k_{2}}\right) \\
& =\sum_{i=0}^{m-1} P\left(G_{k_{1}} \cong \gamma_{i}\right) P\left(\left[Z_{k_{1}}\right] \cong \gamma_{j(i)}, \xi_{k_{2}}=\gamma_{k_{2}}\right)
\end{aligned}
$$

where $\gamma_{i}+\gamma_{j(i)} \cong \gamma_{k_{1}}$, and the last equality is due to the fact that $G_{k_{1}}$ is independent of $\left[Z_{k_{1}}\right]$ and of $\xi_{k_{2}}$. Notice that when $\gamma_{i}$ runs through $\Gamma_{0}$, so does $\gamma_{j(i)}$, and since $P\left(G_{k_{1}} \cong \gamma_{i}\right)=\frac{1}{m}$, we obtain

$$
\begin{aligned}
P\left(\xi_{k_{1}}=\gamma_{k_{1}}, \xi_{k_{2}}=\gamma_{k_{2}}\right) & =\frac{1}{m} \sum_{i=0}^{m-1} P\left(\left[Z_{k_{1}}\right] \cong \gamma_{j(i)}, \xi_{k_{2}}=\gamma_{k_{2}}\right) \\
& =\frac{1}{m} P\left(\xi_{k_{2}}=\gamma_{k_{2}}\right) \\
& =P\left(\xi_{k_{1}}=\gamma_{k_{1}}\right) P\left(\xi_{k_{2}}=\gamma_{k_{2}}\right) .
\end{aligned}
$$

Now assume $P\left(\xi_{k_{1}}=\gamma_{k_{1}}, \ldots, \xi_{k_{n}}=\gamma_{k_{n}}\right)=\prod_{i=1}^{n} P\left(\xi_{k_{i}}=\gamma_{k_{i}}\right)$. Consider

$$
\begin{aligned}
& P\left(\xi_{k_{1}}=\gamma_{k_{1}}, \ldots, \xi_{k_{n}}=\gamma_{k_{n}}, \xi_{k_{n+1}}=\gamma_{k_{n+1}}\right) \\
& \quad=P\left(\xi_{k_{1}}=\gamma_{k_{1}}, \ldots, G_{k_{n}}+\left[Z_{k_{n}}\right] \cong \gamma_{k_{n}}, \xi_{k_{n+1}}=\gamma_{k_{n+1}}\right) \\
& \quad=\sum_{i=0}^{m-1} P\left(\xi_{k_{1}}=\gamma_{k_{1}}, \ldots, G_{k_{n}} \cong \gamma_{i},\left[Z_{k_{n}}\right] \cong \gamma_{j(i)}, \xi_{k_{n+1}}=\gamma_{k_{n+1}}\right),
\end{aligned}
$$

where $\gamma_{i}+\gamma_{j(i)} \cong \gamma_{k_{n}}$. Now, $G_{k_{n}}$ is independent of $\left[Z_{k_{n}}\right]$ and of $\xi_{k_{n+1}}$; by the inductive hypothesis, $G_{k_{n}}$ is also independent of $\xi_{k_{1}}, \ldots, \xi_{k_{n-1}}$. Thus

$$
\begin{aligned}
& \sum_{i=0}^{m-1} P\left(\xi_{k_{1}}=\gamma_{k_{1}}, \ldots, G_{k_{n}} \cong \gamma_{i},\left[Z_{k_{n}}\right] \cong \gamma_{j(i)}, \xi_{k_{n+1}}=\gamma_{k_{n+1}}\right) \\
& =\sum_{i=0}^{m-1} P\left(G_{k_{n}} \cong \gamma_{i}\right) P\left(\xi_{k_{1}}=\gamma_{k_{1}}, \ldots,\left[Z_{k_{n}}\right] \cong \gamma_{j(i)}, \xi_{k_{n+1}}=\gamma_{k_{n+1}}\right) \\
& =\frac{1}{m} \sum_{i=0}^{m-1} P\left(\xi_{k_{1}}=\gamma_{k_{1}}, \ldots,\left[Z_{k_{n}}\right] \cong \gamma_{j(i)}, \xi_{k_{n+1}}=\gamma_{k_{n+1}}\right) \\
& =\frac{1}{m} P\left(\xi_{k_{1}}=\gamma_{k_{1}}, \ldots, \xi_{k_{n-1}}=\gamma_{k_{n-1}}, \xi_{k_{n+1}}=\gamma_{k_{n+1}}\right) \\
& =P\left(\xi_{k_{n}}=\gamma_{k_{n}}\right) \prod_{i \neq n, i=1}^{n+1} P\left(\xi_{k_{i}}=\gamma_{k_{i}}\right)
\end{aligned}
$$

by the inductive hypothesis. So by induction, we have shown that $\left\{\xi_{k}\right\}_{k=1}^{\infty}$ is an independent sequence.

Remark. Theorem 2 is symmetric in $[Z]$ and $G$, but Theorem 3 is not; that is, if $P\left(\left[Z_{k}\right]=\gamma\right)=\frac{1}{m}$ for all $\gamma \in \Gamma_{0}$ but $P\left(G_{k}=\gamma_{0}\right)>\frac{1}{m}$ for some $\gamma_{0} \in \Gamma_{0}$, the 
sequence $\left\{\xi_{k}\right\}_{k=1}^{\infty}$ is not necessarily independent. This is illustrated in the following example: $M=2, \Gamma=\mathbf{Z}$ (the integers) and $\Gamma_{0}=\{0,1\}$. Let $G$ be such that $P(G=0)=P(G=1)=P(G=2)=\frac{1}{3}$. So $P(G \cong 0)=P(G$ is even $)=\frac{2}{3}$ and $P(G \cong 1)=P(G$ is odd $)=\frac{1}{3}$. Then we have

$$
\begin{aligned}
P\left(\left[Z_{1}\right] \text { even }\right) & =P\left(0 \leq Z_{1}<1\right)+P\left(Z_{1}=2\right) \\
& =P\left(G_{1}=0\right)+P\left(G_{1}=1, G_{2}=0\right)+\cdots \\
& =\frac{1}{3} \sum_{k=0}^{\infty}\left(\frac{1}{3}\right)^{k}=\frac{1}{2} .
\end{aligned}
$$

Therefore $\xi_{1}$ is uniform on $\Gamma_{0}$ by Theorem 2 However, the sequence $\left\{\xi_{k}\right\}_{k=1}^{\infty}$ is not independent. Consider $P\left(\xi_{1}=0, \xi_{2}=0\right)$ :

$$
\begin{aligned}
P\left(\xi_{1}\right. & \left.=0, \xi_{2}=0\right) \\
& =P\left(G_{1}+\left[Z_{1}\right] \cong 0, \xi_{2}=0\right) \\
& =P\left(G_{1} \cong 0,\left[Z_{1}\right] \cong 0, \xi_{2}=0\right)+P\left(G_{1} \cong 1,\left[Z_{1}\right] \cong 1, \xi_{2}=0\right) \\
& =\frac{2}{3} P\left(\left[Z_{1}\right] \cong 0, \xi_{2}=0\right)+\frac{1}{3} P\left(\left[Z_{1}\right] \cong 1, \xi_{2}=0\right)
\end{aligned}
$$

To compute the two remaining probabilities, note that

$$
\left[Z_{1}\right]=\left[\sum_{k=1}^{\infty} 2^{-k} G_{k+1}\right]=\left[\frac{G_{2}+\left[Z_{2}\right]}{2}+\frac{\left(Z_{2}\right)}{2}\right]
$$

If $\xi_{2}=0, G_{2}+\left[Z_{2}\right]$ is even, so in this case, $\left[Z_{1}\right]=\frac{G_{2}+\left[Z_{2}\right]}{2}$, and if $\left[Z_{1}\right]=\frac{G_{2}+\left[Z_{2}\right]}{2}$, then $\xi_{2}=0$. This implies that

$$
\begin{aligned}
& \frac{2}{3} P\left(\left[Z_{1}\right] \cong 0, \xi_{2}=0\right)+\frac{1}{3} P\left(\left[Z_{1}\right] \cong 1, \xi_{2}=0\right) \\
&=\frac{2}{3} P\left(\left[Z_{1}\right] \cong 0,\left[Z_{1}\right]=\frac{G_{2}+\left[Z_{2}\right]}{2}\right)+\frac{1}{3} P\left(\left[Z_{1}\right] \cong 1,\left[Z_{1}\right]=\frac{G_{2}+\left[Z_{2}\right]}{2}\right) \\
&=\frac{2}{3} P\left(G_{2}+\left[Z_{2}\right] \cong 0 \bmod 4\right)+\frac{1}{3} P\left(G_{2}+\left[Z_{2}\right] \cong 2 \bmod 4\right) \\
&=\frac{2}{3}\left(P\left(G_{2}=0,\left[Z_{2}\right]=0\right)+P\left(G_{2}=2,\left[Z_{2}\right]=2\right)\right) \\
&+\frac{1}{3}\left(P\left(G_{2}=0,\left[Z_{2}\right]=2\right)+P\left(G_{2}=2,\left[Z_{2}\right]=0\right)+P\left(G_{2}=1,\left[Z_{2}\right]=1\right)\right) \\
&= \frac{2}{3}\left(\frac{1}{2} \cdot \frac{1}{3}+0\right)+\frac{1}{3}\left(0+\frac{1}{3} \cdot \frac{1}{2}+\frac{1}{3} \cdot \frac{1}{2}\right) \\
&= \frac{2}{9} \neq \frac{1}{4}=P\left(\xi_{1}=0\right) P\left(\xi_{2}=0\right) .
\end{aligned}
$$

Since the sequence $\left\{\xi_{k}\right\}_{k=1}^{\infty}$ is not independent, by Theorem 1, $£(Z)$ is not absolutely continuous with respect to Lebesgue measure for this example. Thus, the assumption that $\xi_{1}$ is uniform does not necessarily imply the independence of the sequence $\left\{\xi_{k}\right\}_{k=1}^{\infty}$.

If the range of $G$ is $\Gamma_{0}$, then $[Z]=0$. In this case $G=\xi_{1}$ and the application of Theorem 3 yields the following: 
Corollary 2. Suppose that the range of $G$ is $\Gamma_{0}$. Then

$$
\varphi(x)=\sum_{\gamma \in \Gamma_{0}} c(\gamma) \varphi(M x-\gamma) .
$$

has a functional solution if and only if $P(G=\gamma)=c(\gamma)=\frac{1}{m}$.

The result of Corollary 2 is known. It was first proved by Grochenig and Madych (Theorem 2 in [5]) using different methods. The solution of the dilation equation in this case is $\varphi=\frac{1}{\sqrt{\lambda(T)}} 1_{T}$. Scaling functions that are indicator functions over the tile are used to construct "Haar-type" wavelet bases as discussed in detail in [5].

\section{EXAMPLES}

In this section we give several examples of density functions obtained by assigning probabilities so that the hypotheses of Theorem 3 are satisfied.

In most cases, there is no closed form for the density function [14; those which cannot be computed explicitly can be numerically approximated by computing the function values on the points of $\left\{M^{-k} \Gamma \mid k=0, \ldots, k_{0}\right\}$ for some $k_{0}$, via the dilation equation. To obtain the approximation of the graph of $\varphi$, first the values of $\varphi$ at the integers are found by considering the vector of integer values as an eigenvector of eigenvalue 1 for a matrix of coefficients [14. Then, using the scaling relation (1.1), the values of $\varphi$ can be found at all points in $M^{-1} \Gamma$. Repeatedly applying (1.1) $k_{0}$ times and plotting the results gives an approximation to the graph of $\varphi$. Questions of convergence of the approximations are discussed in [3].

For each of the following examples, the eigenvalue problem for a matrix corresponding to a set containing the support of $\varphi$ was solved to obtain the values at the lattice points. Then the above algorithm was applied, resulting in approximately 2000 points plotted for each graph approximation.

Example 1. Let $d=1, M=2, \Gamma=\mathbf{Z}$ and $\Gamma_{0}=\{0,1\}$. Suppose the range of $G$ is $\Gamma_{1}=\{0,1,2,3\}$ with the following weight assignments: $c(0)=.2, c(1)=.4$, $c(2)=.3, c(3)=.1$. Then the density function $\varphi$ is continuous [2] and is pictured in Figure 1 along with a four-coefficient spline function for comparison.

Example 2. Suppose $d=2, \Gamma=\mathbf{Z}^{2}, \Gamma_{0}=\{(0,0),(1,0),(0,1),(1,1)\}$ and

$$
M=\left[\begin{array}{ll}
2 & 0 \\
0 & 2
\end{array}\right] \text {. }
$$

Define $G$ to have values in $\Gamma_{1}=[0,2]^{2} \cap \mathbf{Z}^{2}$ with the following probability distribution:

$$
\begin{aligned}
& c((0,0))=c((2,0))=c((0,2))=c((2,2))=\frac{1}{16}, \\
& c((1,0))=c((0,1))=c((2,1))=c((1,2))=\frac{1}{8}, \\
& c((1,1))=\frac{1}{4} .
\end{aligned}
$$

Since $G$ is clearly the convolution of two independent copies of a uniform random variable on the unit square, $\varphi$ is continuous. The graph of the density function is pictured in Figure 2. 


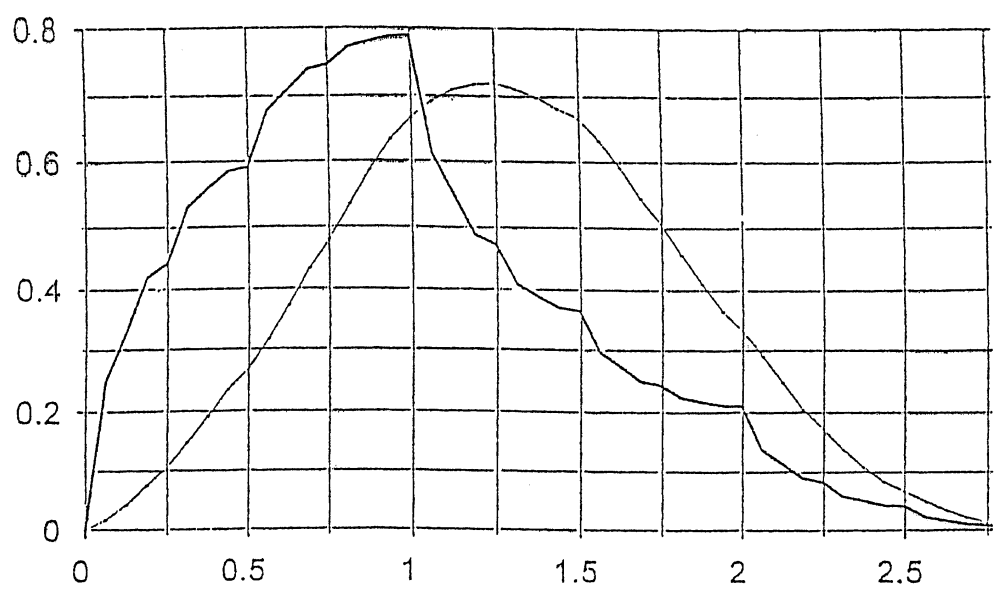

Figure 1.

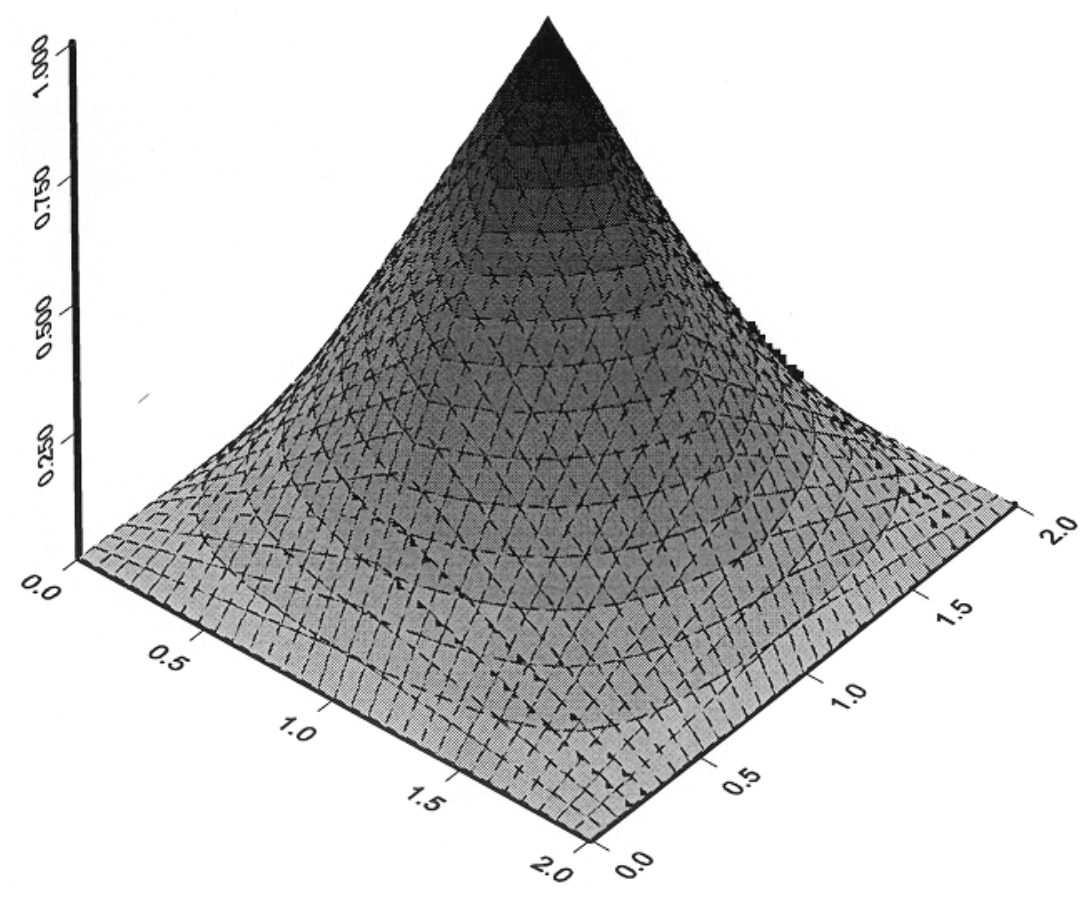

Figure 2.

Example 3. Let $d=2, M=\left[\begin{array}{cc}1 & -1 \\ 1 & 1\end{array}\right], \Gamma=\mathbf{Z}^{2}$ and $\Gamma_{0}=\{(0,0),(1,0)\}$. Define $G$ to have values in $\Gamma_{1}=\{(0,0),(1,0),(2,0)\}$ with the following distribution: $c((0,0))=\frac{1}{4}, c((1,0))=\frac{1}{2}, c((2,0))=\frac{1}{4}$. The graph of the density function is pictured in Figure 3. The density is a convolution of two indicator functions of the twin dragon tile and therefore it is continuous. 


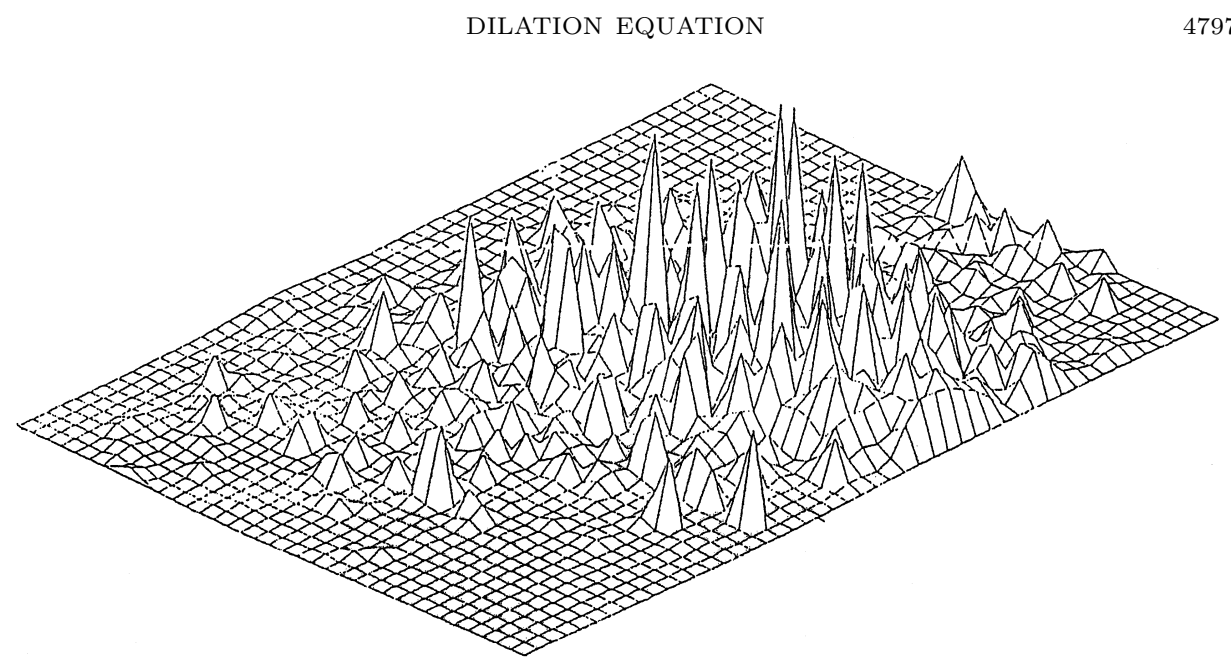

FiguRE 3.

\section{A NECESSARY CONDITION FOR MULTIDIMENSIONAL PRESCALE FUNCTIONS}

Suppose $\varphi$ is a functional solution of the dilation equation (1.1). If the lattice translates of $\varphi$ form a Riesz basis, that is, for some positive constants $C_{1}, C_{2}$

$$
C_{1} \sqrt{\sum(a(\gamma))^{2}} \leq\left\|\sum a(\gamma) \varphi(\cdot-\gamma)\right\|_{L^{2}\left(\mathbf{R}^{d}\right)} \leq C_{2} \sqrt{\sum(a(\gamma))^{2}}
$$

then $\varphi$ is said to be stable. We show that the condition $\sum_{\gamma \cong \delta} c(\gamma)=\frac{1}{m}$ for each $\delta \in \Gamma_{0}$, where $m=|\operatorname{det} M|$, which was sufficient for the existence of a functional solution to (1.1), is necessary for the stability of $\varphi$.

The Fourier transform version of the dilation equation (1.1) is

$$
\widehat{\varphi}(\zeta)=\widehat{\varphi}\left(M^{*-1} \zeta\right) A\left(M^{*-1} \zeta\right)
$$

where $A(\zeta)=\sum_{\gamma \in \Gamma} c(\gamma) e^{-i \gamma \cdot \zeta}$. Stability of $\varphi$ is equivalent to

$$
0<C_{1} \leq \sum_{k \in \Gamma}|\widehat{\varphi}(\zeta+2 \pi k)|^{2} \leq C_{2} \text { a.e. }
$$

In the case that the coefficient sequence $c:=\{c(\gamma)\}_{\gamma \in \Gamma}$ is finitely supported, the function in (6.2) is a polynomial [12] and so the inequality must hold everywhere. In the theorem below, which is known (see, for example, [9]), we will assume that the equation holds everywhere. This is not a restriction as proved in 44. For completeness we include a short proof.

Theorem 4. Let $\varphi \in L^{2}\left(\mathbf{R}^{d}\right)$ be a solution of the dilation equation (1.1). Suppose $\varphi$ is stable and that equation (6.2) holds everywhere. Then $\sum_{\gamma \cong \gamma_{0}} c(\gamma)=\frac{1}{m}$ for each $\gamma_{0} \in \Gamma_{0}$. 
Proof. Without loss of generality, we assume $\Gamma=\mathbf{Z}^{d}$. Since $\varphi$ is stable, (6.2) holds. Applying equation (6.1) we obtain

$$
\begin{aligned}
0 & <C_{1} \leq \sum_{k \in \mathbf{Z}^{d}}|\widehat{\varphi}(\zeta+2 \pi k)|^{2} \\
& =\sum_{\gamma \in \Gamma_{0}}\left|A\left(M^{*-1} \zeta+2 \pi M^{*-1} \gamma\right)\right|^{2} \sum_{k^{\prime} \in \mathbf{Z}^{d}}\left|\widehat{\varphi}\left(M^{*-1} \zeta+2 \pi\left(M^{*-1} \gamma+k^{\prime}\right)\right)\right|^{2}
\end{aligned}
$$

For $\zeta=0$, we get

$$
\begin{aligned}
\sum_{k \in \mathbf{Z}^{d}}|\widehat{\varphi}(2 \pi k)|^{2}= & \sum_{\gamma \in \Gamma_{0} \backslash\{0\}}\left|A\left(2 \pi M^{*-1} \gamma\right)\right|^{2} \sum_{k^{\prime} \in \mathbf{Z}^{d}}\left|\widehat{\varphi}\left(2 \pi\left(M^{*-1} \gamma+k^{\prime}\right)\right)\right|^{2} \\
& +|A(0)|^{2} \sum_{k^{\prime} \in \mathbf{Z}^{d}}\left|\widehat{\varphi}\left(2 \pi k^{\prime}\right)\right|^{2} .
\end{aligned}
$$

Since by [3] $A(0)=1$, and since $\sum_{k^{\prime} \in \mathbf{Z}^{d}}\left|\widehat{\varphi}\left(2 \pi\left(M^{*-1} \gamma+k^{\prime}\right)\right)\right|^{2} \geq C_{1}>0$, we have

$$
\sum_{k \in \mathbf{Z}^{d}} c(k) e^{-i 2 \pi\left(M^{*-1} \gamma\right) \cdot k}=0 \text { for each } \gamma \in \Gamma_{0} \backslash\{0\},
$$

which, after letting $k=\gamma_{k}+M n_{k}, \gamma_{k} \in \Gamma_{0}, n_{k} \in \mathbf{Z}^{d}$ and setting $\sum_{k \cong \delta} c\left(\gamma_{k}+M n_{k}\right)=$ $q_{\delta}$ leads to

$$
0=\sum_{\delta \in \Gamma_{0}} e^{-i 2 \pi \gamma \cdot M^{-1} \delta} q_{\delta}
$$

Claim. $\sum_{\delta \in \Gamma_{0}} e^{-i 2 \pi \gamma \cdot M^{-1} \delta}=0$ for each $\gamma \in \Gamma_{0} \backslash\{0\}$.

Notice that the set $\left\{e^{-i 2 \pi \gamma \cdot M^{-1}} \delta \mid \delta \in \Gamma_{0}\right\}$ is a group on the unit circle. If

$$
\sum_{\delta \in \Gamma_{0}} e^{-i 2 \pi \gamma \cdot M^{-1} \delta}=r \neq 0
$$

then for every $\gamma \in \Gamma_{0} \backslash\{0\}$ there is a $\delta \in \Gamma_{0}$ so that $e^{-i 2 \pi \gamma \cdot M^{-1} \delta} \neq 1$. (If not, $e^{-i 2 \pi \gamma \cdot M^{-1} \delta}=1$ for all $\delta \in \Gamma_{0}$ implies that

$$
0=\sum_{\delta \in \Gamma_{0}} \sum_{k: k \cong \delta} c\left(\gamma_{k}+M n_{k}\right)=\sum c(\gamma)
$$

contradicting $\sum c(\gamma)=1$.) Multiplying both sides of (6.4) by $e^{-i 2 \pi \gamma \cdot M^{-1} p}$ where $p \in \Gamma_{0}$ is such that $e^{-i 2 \pi \gamma \cdot M^{-1} p} \neq 1$, we obtain

$$
\sum_{\delta \in \Gamma_{0}} e^{-i 2 \pi \gamma \cdot M^{-1}(\delta+p)}=r e^{-i 2 \pi \gamma \cdot M^{-1} p} .
$$

Note that since $\delta+p=\delta^{\prime}+M k$, where $\delta^{\prime} \in \Gamma_{0}$ and $k \in \mathbf{Z}^{d}$, then $\sum_{\delta \in \Gamma_{0}} e^{-i 2 \pi \gamma \cdot M^{-1}(\delta+p)}$ includes all the elements of the group and nothing more, and therefore it is equal to $r$. So $r=r e^{-i 2 \pi \gamma \cdot M^{-1} p}$, contradicting $e^{-i 2 \pi \gamma \cdot M^{-1} p} \neq 1$. 
The set of $m-1$ equations $\sum_{\delta \in \Gamma_{0}} e^{-i 2 \pi \gamma \cdot M^{-1} \delta} q_{\delta}=0$ for each $\gamma \in \Gamma_{0} \backslash\{0\}$, along with the constraint $\sum_{\delta \in \Gamma_{0}} q_{\delta}=1$, comprises a system of $m$ equations with $m$ variables $q_{\delta}$. Notice that $q_{\delta}=\frac{1}{m}$ for each $\delta \in \Gamma_{0}$ is a solution. The coefficient matrix for this system is given by

$$
U=\left(e^{-i 2 \pi \gamma_{i} \cdot M^{-1} \gamma_{j}}\right)_{0 \leq i, j \leq m-1}
$$

By (6.3) $), U U^{*}=m I_{m}$, and so $\operatorname{det} U \neq 0$. Therefore $q_{\delta}=\frac{1}{m}$ for all $\delta \in \Gamma_{0}$ is the unique solution of the system, which concludes the proof of the theorem.

If, as in the previous section, we let $c(\gamma)=P(G=\gamma)$, the above theorem says that $P(G \cong \gamma)=\frac{1}{m}$ for each $\gamma \in \Gamma_{0}$ is necessary in order for the density $\varphi$ to be stable. However, this condition, which by Theorems 1 and 3 guarantees that $\varphi$ is absolutely continuous, is not sufficient for the stability of $\varphi$. Consider the following example: $\Gamma=\mathbf{Z}, M=2$ with the constants assigned as follows:

$$
\begin{aligned}
& c(0)=c(2)=c(3)=c(5)=\frac{1}{8}, \\
& c(1)=c(4)=\frac{1}{4} .
\end{aligned}
$$

Notice that the two cosets have equal weight and so by Theorems 1 and 3 the solution $\varphi$ of the dilation equation will be a density function. However, it is shown in [1] that $\varphi$ is not stable.

\section{REFERENCES}

1. C. Bandt, Self-similar sets. V. Integer matrices and fractal tilings of $R^{n}$, Proc. Amer. Math. Soc., 112, (2) (1991), pp. 549-562. MR 92d:58093

2. D. Colella and C. Heil, Dilation equations and the smoothness of compactly supported wavelets in Wavelets: Mathematics and Applications (J. Benedetto and M. Frazier, eds.), CRC Press, Boca Raton, FL, 1994, pp. 163-201. MR 94j:42049

3. I. Daubechies, Ten Lectures on Wavelets, CMBS Regional Conference Series in Applied Mathematics, Vol. 61, SIAM, Philadelphia, PA, 1992. MR 93e:42045

4. V. Dobric, R.F. Gundy, P. Hitczenko, Characterizations of orthonormal scale functions: $a$ probabilistic approach, J. Geom. Anal. 10 (2000), pp. 417-434. CMP 2001:04

5. K. Gröchenig and W. R. Madych, Multiresolution analysis, Haar bases and self-similar tilings of $\mathbf{R}^{n}$, IEEE Trans. Inform. Theory, 38 (2) (1992), pp. 556-567. MR 93i:42001

6. R. Gundy and C. Zhang, "Dilation equations," Lehigh University Probability Seminar, Fall, 1994.

7. J. Hoffmann-Jørgensen, Measures which agree on balls, Math. Scand., 37 (1975), pp. 319-326. MR 53:13509

8. B. Jessen and A. Wintner, Distribution functions and the Riemann zeta function, Trans. Amer. Math. Soc., 38 (1935), pp. 44-88. CMP 95:18

9. Q. Jiang, Multivariate matrix refinable functions with arbitrary matrix dilation, Trans. Amer. Math. Soc., 351 (6) (1999), pp. 2407-2438. MR 99i:42047

10. J. Lagarias and Y. Wang, Self-Affine Tiles in $\mathbf{R}^{n}$, Adv. Math., 121 (1996), pp. 21-49. MR 97d:52034

11. W. Lawton, S. L. Lee, Z. Shen, Stability and orthonormality of multivariate refinable functions, SIAM J. Math. Anal., 28 (4) (1997), pp. 999-1014. MR 98d:41027

12. P. G. Lemarie, Fonctions a support compact dans les analyses multi-resolutions, Rev. Mat. Iberoamericana, 7 (2) (1991), pp. 157-182. MR 93b:42046 
13. C. A., Rogers, Analytic Sets, Academic Press, New York, 1980.

14. G. Strang, Wavelet transforms versus Fourier transforms, Bull. Amer. Math. Soc., 28 (2) (1993), pp. 288-305. MR 94b:42017

Department of Mathematics, West Chester University of Pennsylvania, West Chester, Pennsylvania 19383

Current address: Department of Mathematics, Salem State College, Salem, Massachusetts 01970

E-mail address: jbelock@salemstate.edu

Department of Mathematics, Lehigh University, 14 Packer Avenue, Bethlehem, PennSYLVANIA 18015

E-mail address: vd00@lehigh.edu

URL: http://www.lehigh.edu/ vd00/vd00.html 